УДК 550.4.08

\title{
СРАВНЕНИЕ ИСП-МС АНАЛИЗА ГЕОЛОГИЧЕСКИХ ОБРАЗЦОВ В ВАРИАНТЕ РАСТВОРОВ И ЛАЗЕРНОЙ АБЛЯЦИИ СТЕКОЛ
}

\author{
Николаева Ирина Викторовна', \\ iniko@igm.nsc.ru
}

Палесский Станислав Владиславович',
stas@igm.nsc.ru

Карпов Александр Викторович',

theblake@mail.ru

Институт геологии и минералогии им. В.С. Соболева СО РАН,
Россия, г. Новосибирск, 630090, пр. Академика Коптюга, 3.

Актуальность исследования связана с необходимостью опробования нового эффективного способа пробоподготовки геологических образцов, подходящего для массового многоэлементного ИСП-МС анализа, снижающего общие временные затраты и обеспечивающего возможность определения широкого набора элементов в рамках одного измерительного цикла с применением лазерной абляции (ЛА-ИСП-МС).

Цель исследования состоит в сравнении метрологических характеристик двух методик ИСП-МС анализа, основанных на разных способах пробоподготовки силикатных пород - переведении твердого образца в раствор после сплавления с метаборатом лития и использовании готовых боратных стекол для ЛА-ИСП-МС анализа.

Объекты: международные и отечественные стандартные образцы состава естественных горных пород: базальты - ВНVО-1, BHVO-2, BCR-2, граниты - СГ-1а, СГ-3, габбро СГД-1а, трапп CT-1а.

Методы: масс-спектрометрия с индуктивно-связанной плазмой (ИСП-МС), лазерная абляция с масс-спектрометрическим окончанием (ЛА-ИСП-МС).

Результаты исследования показали, что разработанные методики многоэлементного ИСП-МС И ЛА-ИСП-МС анализа позволяют определять широкий набор элементов в геологических образцах в рамках одного измерительного цикла в высоком, среднем и низком разрешении при использовании для внешней градуировки стандартных образцов с аналогичной матрицей и внутреннего стандарта для учета и снижения матричного влияния и дрейфа приборных параметров. Правильность определения всех элементов в соответствии с разработанными методиками доказана сравнением результатов анализа шести стандартных геологических образцов с принятыми значениями. ЛА-ИСП-МС отличается эКспрессностью за счет отсутствия стадии пробоподготовки, при этом пределы обнаружения и погрешность определения для ЛА-ИСП-МС методики выше, чем при ИСП-МС анализе растворов.

\section{Ключевые слова:}

Масс-спектрометрия с индуктивно-связанной плазмой (ИСП-МС), лазерная абляция (ЛА), пробоподготовка, стандартные геологические образцы, основные и примесные элементы, редкоземельные элементы.

\section{Введение}

Одной из важнейших аналитических задач в области наук о Земле является определение состава пород и минералов. Всестороннее описание элементного состава геологических пород и минералов до недавнего времени требовало применения в комплексе нескольких аналитических методов, т. к. ни один метод не позволяет одновременно определять весь набор элементов, интересующих геохимиков и геологов, или из-за недостаточно низких пределов обнаружения, или из-за ограничения по набору элементов.

Внедрение в аналитическую практику современного метода - масс-спектрометрии с индуктивно-связанной плазмой (ИСП-МС) - существенно расширяет возможности элементного анализа за счет одновременного определения практически всех химических элементов с низкими пределами обнаружения в рамках одного измерения с широким линейным диапазоном определяемых концентраций. Использование различных устройств для получения аэрозоля пробы позволяет анализировать как растворы, так и твердые образцы [1-5].
Основным требованием ИСП-МС анализа в варианте растворов является полный перевод определяемых элементов в раствор, обеспечение устойчивости растворов и снижение содержания матричных элементов. Именно стадия химической пробоподготовки, определяющая правильность всего анализа в целом, является самой сложной и продолжительной, особенно для трудновскрываемых геологических образцов, поэтому остаётся актуальным поиск альтернативных способов подготовки образцов к анализу.

Использование специальной лазерной установки для проведения лазерной абляции (ЛА) в сочетании с ИСП масс-спектрометром позволяет выполнять анализ твердых образцов (ЛА-ИСП-МС) без длительной стадии переведения определяемых элементов в раствор. Обычно ЛА-ИСП-МС используют для локального анализа мономинеральных фракций [6-8], однако существуют различные подходы, которые позволяют определять валовый элементный состав пород [9-18]. Наиболее простым и эффективным способом подготовки твердых образцов для ЛА-ИСП-МС представляется ис- 
пользование стекол, уже приготовленных для рентгено-флуоресцентного анализа (РФА) сплавлением навески измельчённого образца с мета- или тетработатом лития в разных соотношениях. Эта процедура позволяет полностью переводить исследуемую породу в боратное стекло, при этом достигается вскрытие акцессорных минералов и гомогенизация образца. Разбавление образца в 2-10 раз мета- или тетраборатом лития уменьшает взаимное влияние элементов при ИСП-МС измерениях. В качестве минусов такого способа подготовки проб можно отметить загрязнение образца материалом флюса и тигля, повышение пределов обнаружения за счет разбавления [9-14].

В сравнении с ИСП-МС анализом в варианте растворов такой способ подготовки образцов снимает проблемы нестабильности растворов и существенно увеличивает скорость ИСП-МС анализа, а в сочетании с РФА обеспечивает расширение набора определяемых элементов из одной навески без дополнительных стадий химической подготовки. Однако при ЛА-ИСП-МС анализе твёрдых образцов наблюдается фракционирование элементов, возникающее при генерации аэрозоля, его транспортировке, атомизации и ионизации в плазме $[5,10$, $14,19]$. В связи с этим для получения достоверных результатов при ЛА-ИСП-МС анализе необходимо использовать градуировочные стандарты с идентичной матрицей, а также проводить нормализацию каждого значения с помощью внутреннего стандарта - элемента, входящего в состав анализируемого образца и определенного независимым методом. Это необходимо для коррекции возможной разницы в образовании и транспортировке аэрозоля анализируемого и градуировочного образца в зависимости от характеристик самих образцов и параметров лазерного излучения $[5,10,14,19]$.

Целью настоящей работы является изучение возможностей ЛА-ИСП-МС анализа стекол, приготовленных для РФА, с определением максимального количества элементов в рамках одного измерительного цикла и сравнение метрологических характеристик этой методики с ИСП-МС анализом растворов.

\section{Экспериментальная часть. Объекты анализа}

В настоящей работе в качестве объектов анализа были использованы международные стандартные геологические образцы: базальты - BHVO-1, BHVO-2, BCR-2, и отечественные стандартные образцы состава естественных горных пород: граниты - СГ-1а, СГ-3, габбро СГД-1а, трапп СТ-1а. Анализируемые образцы характеризуются разным уровнем содержания определяемых элементов и аттестованы с различной точностью. Для некоторых элементов в литературе указаны рекомендованные значения без погрешности ввиду недостаточного количества аналитических данных. Поэтому далее в тексте для общего обозначения аттестованных и рекомендованных значений используется термин «принятые значения».
Для перевода в раствор твердых образцов использовали методику сплавления с метаборатом лития с последующим растворением плава в $5 \%$ азотной кислоте со следовыми количествами фтористоводородной кислоты [20].

Для ЛА-ИСП-МС анализа мы использовали уже готовые стёкла. Они были приготовлены в лаборатории рентгеноспектральных методов анализа Института геологии и минералогии СО РАН по следующей методике. Анализируемую пробу сушили при $105^{\circ} \mathrm{C}$ в течение 1 часа, затем прокаливали при $1000{ }^{\circ} \mathrm{C}$ в течение 2,5 часов, после чего смешивали с флюсом (66,67 \% тетрабората лития; $32,83 \%$ метабората лития и 0,5 \% лития бромистого) в соотношении 1:9 (общий вес смеси составляет 5 г). Смесь плавили в платиновых тиглях в индукционной печи Lifumat-2,0-0x (Linn High Therm Gmbh). В качестве контрольного опыта использовали боратное стекло, плавленое без образца [21].

\section{ИСП-МС измерения}

Все измерения выполнены на ИСП масс-спектрометре высокого разрешения ELEMENT фирмы Finnigan Mat (Германия) в аналитическом центре Института геологии и минералогии им. В.С. Соболева СО РАН.

Перед измерениями проводили настройку прибора и оптимизацию инструментальных параметров для получения максимально интенсивного и хорошо воспроизводимого сигнала при низком фоновом уровне шума и сигналов двухзарядных и оксидных ионов [20]. Сканирование проводили в интервале 23-238 атомных единиц массы в режиме «peak jumping» и, в зависимости от определяемого элемента, в низком $(\mathrm{M} / \Delta \mathrm{M}=300)$, среднем (4000) или высоком (8000) разрешении. Каждый массовый диапазон сканировали 40 раз. Детектирование велось в аналоговом и цифровом режиме (analog+counting).

При анализе сплавленных стёкол использовали установку для ЛА - UP-213 фирмы New Wave Research с ультрафиолетовым лазером $\mathrm{Nd}: \mathrm{YAG}$ (длина волны 213 нм). В качестве газа-носителя был выбран Не, поскольку он обеспечивает более высокую эффективность транспортировки аэрозоля в плазму и меньшее фракционирование элементов в сравнении с $\operatorname{Ar}[8,10]$. Перед каждым измерением ячейку абляции продували потоком Не, чтобы избежать попадания воздуха в плазму. Перед введением в плазму Не (скорость потока 0,2 мл/мин) смешивали с Ar в соотношении 1:4. Для проведения абляции диаметр пучка лазера был выбран 80 мкм, плотность мощности лазерного излучения $2 \cdot 10^{8} \mathrm{~B}$ т $\mathrm{cm}^{2}$, частота импульсов 20 Гц, продолжительность импульса 5 нс.

\section{Определяемые элементы и изотопы, градуировка}

Набор определяемых элементов для растворов и сплавленных стекол составил 43 элемента, включая как основные элементы, так и примес- 
ные: $\mathrm{Al}, \mathrm{Ba}, \mathrm{Ca}, \mathrm{Ce}, \mathrm{Co}, \mathrm{Cr}, \mathrm{Cs}, \mathrm{Cu}, \mathrm{Dy}, \mathrm{Eu}, \mathrm{Er}, \mathrm{Fe}$, $\mathrm{Ga}, \mathrm{Gd}, \mathrm{Hf}, \mathrm{Ho}, \mathrm{K}, \mathrm{La}, \mathrm{Lu}, \mathrm{Mg}, \mathrm{Mn}, \mathrm{Na}, \mathrm{Nb}, \mathrm{Nd}, \mathrm{Ni}$, P, Pr, Rb, Sc, Si, Sm, Sr, Ta, Tb, Th, Ti, Tm, U, V, Y, $\mathrm{Yb}, \mathrm{Zn}, \mathrm{Zr}$.

В качестве аналитов были выбраны наиболее распространенные изотопы, свободные от изобарных наложений. При наличии нескольких изотопов определение вели по 2-3 изотопам для учета возможных наложений, как и при анализе растворов [20, 22]. Изотоп ${ }^{39} \mathrm{~K}$ определяли в высоком разрешении из-за существенных наложений ${ }^{38} \mathrm{Ar}^{1} \mathrm{H}^{+}$. Изотопы $\mathrm{Sc}, \mathrm{Si}, \mathrm{P}, \mathrm{Ca}, \mathrm{Ti}, \mathrm{Cr}$ и $\mathrm{Fe}$ определяли только в среднем разрешении также из-за существенных наложений, которые состоят в основном из макрокомпонентов плазмы - аргона, кислорода, водорода, азота и основных компонентов пробы. Изотопы $\mathrm{Na}, \mathrm{Mg}, \mathrm{Al}, \mathrm{V}, \mathrm{Mn}, \mathrm{Ni}, \mathrm{Cu}$, и Zn определяли как в среднем, так и в низком разрешении, поскольку наложения на них могут быть несущественными. Остальные элементы от Rb до U определяли только в низком разрешении, в этой области масс спектральные помехи от оксидных и двухзарядных ионов примесных элементов для изученных образцов незначительны, за исключением наложения оксидов бария на изотопы европия [20, 22].

При расчете концентраций использовали усредненные значения, полученные по разным изотопам и в разном разрешении, при отсутствии существенных различий. Расчет концентраций при анализе растворов выполняли по внешней градуировке с внутренним стандартом, в качестве которого выбран In с концентрацией в растворе 1 мкг/л. Для внешней градуировки, как и в работах [20, 22], использовали стандартный образец BHVO-1, наиболее полно и точно аттестованный.

При анализе сплавленных стёкол при помощи ЛА-ИСП-МС использовали внешнюю градуировку по стандартному образцу - BHVO-2, приготовленному по той же методике, что и все анализируемые образцы. В качестве внутреннего стандарта использовали аттестованные значения концентрации Fe.

\section{Результаты и обсуждение. Пределы обнаружения определяемых элементов с использованием ИСП-МС и ЛА-ИСП-МС методик}

В табл. 1 представлены достигнутые пределы обнаружения ИСП-МС и ЛА-ИСП-МС методик в пересчёте на твердый образец, с учетом навески и разбавления, рассчитанные по $3 \sigma$ вариации контрольного опыта, который включал в себя все стадии пробоподготовки, что и анализируемые образцы.

Различие пределов обнаружения для определяемых элементов связано с разницей в чувствительности, которая растет с увеличением атомной массы иона, а также с уровнем контрольного опыта и используемым разрешением (при переходе от низкого разрешения к среднему чувствительность уменьшается примерно на порядок, а при переходе от среднего к высокому - в 3-5 раз). Пределы обна- ружения для элементов, концентрации которых в геологических образцах велики $(\mathrm{Na}, \mathrm{Mg}, \mathrm{Al}, \mathrm{Si}, \mathrm{K}$, $\mathrm{Ca}, \mathrm{Fe})$, дополнительно увеличиваются за счёт «эффекта памяти», возникшего в результате многолетних анализов геологических образцов.

Таблииа 1. Предель обнаружения $\left(C_{\min }\right)$, мкг/г

Table 1. Detection limits $\left(C_{\min }\right), p p m$

\begin{tabular}{|c|c|c|c|c|c|c|c|c|}
\hline 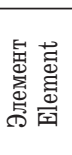 & 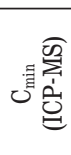 & 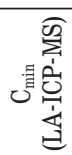 & 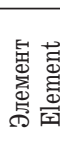 & 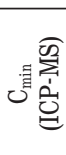 & & 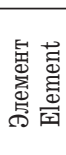 & 记是 & ن \\
\hline $\mathrm{Na}$ & 200 & 200 & $\mathrm{Cu}$ & 5 & 25 & $\mathrm{Eu}$ & 0,003 & 0,02 \\
\hline $\mathrm{Mg}$ & 50 & 100 & $\mathrm{Zn}$ & 5 & 12 & $\mathrm{Gd}$ & 0,02 &, 06 \\
\hline $\mathrm{Al}$ & 30 & 260 & $\mathrm{Ga}$ & 1 & 1 & $\mathrm{~Tb}$ & 0,01 &, 03 \\
\hline $\mathrm{Si}$ & 400 & 3800 & $\mathrm{Rb}$ & 0,5 & 1 & Dy & 0,01 & 0,03 \\
\hline$P$ & 4 & 400 & $\mathrm{Sr}$ & 3 & 2 & Ho & 0,003 & 0,02 \\
\hline $\mathrm{K}$ & 80 & 100 & $\bar{Y}$ & 0,1 & 0,1 & $\mathrm{Er}$ & 0,02 & 0,15 \\
\hline $\mathrm{Ca}$ & 100 & 300 & $\mathrm{Zr}$ & 0,22 & 2 & $\mathrm{Tm}$ & 0,01 & 0,01 \\
\hline $\mathrm{Sc}$ & 0,1 & 0,05 & $\mathrm{Nb}$ & 0,09 & 0,09 & $\mathrm{Yb}$ & 0,02 & 0,01 \\
\hline $\mathrm{Ti}$ & 10 & 10 & $\mathrm{Cs}$ & 0,1 & 0,5 & $\mathrm{Lu}$ & 0,003 & 0,01 \\
\hline $\mathrm{V}$ & 0,3 & 1 & $\mathrm{Ba}$ & 5,5 & 3 & $\mathrm{Hf}$ & 0,09 & 0,02 \\
\hline $\mathrm{Cr}$ & 3 & 4 & $\mathrm{La}$ & 0,06 & 0,08 & $\mathrm{Ta}$ & 0,15 & 0,02 \\
\hline $\mathrm{Mn}$ & 2 & 1 & $\mathrm{Ce}$ & 0,06 & 0,1 & Th & 0,03 & 0,03 \\
\hline $\mathrm{Fe}$ & 20 & 50 & $\mathrm{Pr}$ & 0,03 & 0,03 & $\mathrm{U}$ & 0,02 & 0,02 \\
\hline Co & 0,1 & 0,1 & $\mathrm{Nd}$ & 0,03 & 0,09 & & & \\
\hline $\mathrm{Ni}$ & 3 & 3 & $\mathrm{Sm}$ & 0,01 & 0,02 & & & \\
\hline
\end{tabular}

Более высокие пределы обнаружения ЛА-ИСП-МС методики для таких элементов, как $\mathrm{Al}, \mathrm{Si}, \mathrm{Cu}$ и в особенности Р, связаны с их высоким содержанием в контрольном опыте. Для большинства остальных элементов пределы обнаружения обеих методик сравнимы.

\section{Результаты ИСП-МС и ЛА-ИСП-МС}

\section{анализа стандартных образцов}

В табл. 2-4 приведены результаты определения элементов в стандартных геологических образцах в варианте растворов (ИСП-МС) и при лазерной абляции стекол (ЛА-ИСП-МС) в сравнении с принятыми значениями [23-25]. При использовании ИСП-МС методики по большинству элементов наблюдается удовлетворительное согласие, как для основных элементов, так и для примесных. Погрешность анализа не превышает $10 \%$, если уровень содержания не приближается к пределу обнаружения.

Для ЛА-ИСП-МС анализа относительное стандартное отклонение результатов больше, чем при анализе растворов, и составляет в среднем около $20 \%$. Необходимо отметить, что содержание Cs в BHVO-2 находится на пределе обнаружения ЛА-ИСП-МС (табл. 1), в связи с этим количественные результаты по Cs в анализируемых образцах невозможно получить при использовании для анализа данного градуировочного стандарта. Наибольшие отклонения от принятых значений отмечаются для элементов, содержание которых близки к пределам обнаружения, например, $\mathrm{Mg}, \mathrm{V}, \mathrm{Ni}$, Ва в СГ-1а (табл. 3). Содержание Р в СГ-1а и СГ-3 
Таблица 2. Результаты ИСП-МС и ЛА-ИСП-МС определения концентраций элелентов в стандартных образиах ВНVО-2 и ВСR-2. Концентрации основных элементов приведены в массовых \%, примесных элементов - в мкг/ح

Table 2. Results of ICP-MS and LA-ICP-MS analyses of reference materials BHVO-2 and BCR-2. Concentrations of major (wt. \%) and trace elements (ppm) are presented

\begin{tabular}{|c|c|c|c|c|c|}
\hline \multirow{2}{*}{$\begin{array}{l}\text { Элемент } \\
\text { Element }\end{array}$} & \multicolumn{2}{|c|}{ BHVO-2 $(\mathrm{n}=15)$} & \multicolumn{3}{|c|}{ BCR-2 $(n=8)$} \\
\hline & $\begin{array}{l}\mathrm{C}_{\text {mean }} \pm \Delta \\
\text { ICP-MS }\end{array}$ & $\begin{array}{l}\text { Принятые значения } \\
\text { Reference value [23] }\end{array}$ & $\begin{array}{l}\mathrm{C}_{\text {mean }} \pm \Delta \\
\text { ICP-MS }\end{array}$ & $\begin{array}{c}\mathrm{C}_{\text {mean }} \pm \Delta \\
\text { LA-ICP-MS }\end{array}$ & $\begin{array}{l}\text { Принятые значения } \\
\text { Reference value [23] }\end{array}$ \\
\hline $\mathrm{Na}$ & $(1,6 \pm 0,1) \%$ & $(1,64 \pm 0,05) \%$ & $(2,5 \pm 0,4) \%$ & $(2,0 \pm 0,4) \%$ & $(2,31 \pm 0,04) \%$ \\
\hline $\mathrm{Mg}$ & $(4,5 \pm 0,4) \%$ & $(4,38 \pm 0,04) \%$ & $(2,1 \pm 0,2) \%$ & $(2,0 \pm 0,5) \%$ & $(2,17 \pm 0,04) \%$ \\
\hline $\mathrm{Al}$ & $(7,1 \pm 0,4) \%$ & $(7,11 \pm 0,06) \%$ & $(6,7 \pm 0,4) \%$ & $(6,4 \pm 0,7) \%$ & $(7,1 \pm 0,1) \%$ \\
\hline $\mathrm{Si}$ & $(22 \pm 1) \%$ & $(23,1 \pm 0,1) \%$ & $(26 \pm 2) \%$ & $(26 \pm 5) \%$ & $(25,2 \pm 0,2) \%$ \\
\hline $\mathrm{P}$ & $(0,12 \pm 0,03) \%$ & $(0,117 \pm 0,005) \%$ & $(0,16 \pm 0,02) \%$ & $(0,13 \pm 0,05) \%$ & $(0,16 \pm 0,01) \%$ \\
\hline $\mathrm{K}$ & $(0,43 \pm 0,02) \%$ & $(0,426 \pm 0,004) \%$ & $(1,4 \pm 0,2) \%$ & $(1,5 \pm 0,4) \%$ & $(1,47 \pm 0,02) \%$ \\
\hline $\mathrm{Ca}$ & $(8,8 \pm 0,5) \%$ & $(8,15 \pm 0,06) \%$ & $(5,0 \pm 0,2) \%$ & $(4,7 \pm 0,2) \%$ & $(5,08 \pm 0,08) \%$ \\
\hline $\mathrm{Ti}$ & $(1,7 \pm 0,2) \%$ & $(1,64 \pm 0,02) \%$ & $(1,4 \pm 0,1) \%$ & $(1,3 \pm 0,3) \%$ & $(1,35 \pm 0,03) \%$ \\
\hline $\mathrm{Mn}$ & $(0,14 \pm 0,02) \%$ & $(0,131 \pm 0,002) \%$ & $(0,15 \pm 0,01) \%$ & $(0,143 \pm 0,04) \%$ & $(0,152 \pm 0,003) \%$ \\
\hline $\mathrm{Fe}$ & $(9 \pm 1) \%$ & $(8,67 \pm 0,09) \%$ & $(9,4 \pm 0,2) \%$ & $9,6^{*}$ & $(9,6 \pm 0,3) \%$ \\
\hline $\mathrm{Sc}$ & $32 \pm 2$ & $31,8 \pm 0,3$ & $33 \pm 3$ & $33 \pm 9$ & $33,5 \pm 0,4$ \\
\hline $\mathrm{V}$ & $324 \pm 30$ & $318 \pm 2$ & $411 \pm 36$ & $391 \pm 70$ & $418 \pm 4$ \\
\hline $\mathrm{Cr}$ & $302 \pm 30$ & $287 \pm 3$ & $16 \pm 2$ & $17 \pm 4$ & $15,8 \pm 0,4$ \\
\hline Co & $49 \pm 3$ & $44,9 \pm 0,3$ & $38 \pm 2$ & $36 \pm 10$ & $37,0 \pm 0,4$ \\
\hline $\mathrm{Ni}$ & $131 \pm 11$ & $120 \pm 1$ & $14 \pm 3$ & $15 \pm 7$ & $12,6 \pm 0,3$ \\
\hline $\mathrm{Cu}$ & $135 \pm 6$ & $129 \pm 1$ & $26 \pm 4$ & $31 \pm 10$ & $19,7 \pm 0,7$ \\
\hline $\mathrm{Zn}$ & $108 \pm 6$ & $104 \pm 1$ & $138 \pm 10$ & $129 \pm 30$ & $130 \pm 2$ \\
\hline $\mathrm{Ga}$ & $20 \pm 1$ & $21,4 \pm 0,2$ & $19 \pm 4$ & $19 \pm 4$ & $22,0 \pm 0,2$ \\
\hline $\mathrm{Rb}$ & $9,0 \pm 0,4$ & $93 \pm 0,1$ & $46 \pm 2$ & $50 \pm 11$ & $46,0 \pm 0,6$ \\
\hline $\mathrm{Sr}$ & $390 \pm 14$ & $394 \pm 21$ & $336 \pm 10$ & $310 \pm 60$ & $337 \pm 7$ \\
\hline $\mathrm{Y}$ & $26,4 \pm 0,9$ & $25,9 \pm 0,3$ & $36 \pm 1$ & $36 \pm 8$ & $36 \pm 0,4$ \\
\hline $\mathrm{Zr}$ & $173 \pm 8$ & $171 \pm 1$ & $187 \pm 6$ & $163 \pm 40$ & $186,5 \pm 1,5$ \\
\hline $\mathrm{Nb}$ & $19 \pm 1$ & $18,1 \pm 0,2$ & $12,1 \pm 0,4$ & $13 \pm 3$ & $12,4 \pm 0,2$ \\
\hline $\mathrm{Cs}$ & $0,13 \pm 0,02$ & $0,0996 \pm 0,002$ & $1,0 \pm 0,1$ & н.о. & $1,1 \pm 0,1$ \\
\hline $\mathrm{Ba}$ & $128 \pm 7$ & $131 \pm 1$ & $670 \pm 30$ & $645 \pm 100$ & $684 \pm 5$ \\
\hline $\mathrm{Hf}$ & $4,1 \pm 0,6$ & $4,47 \pm 0,02$ & $4,8 \pm 0,3$ & $4,8 \pm 1,1$ & $4,97 \pm 0,03$ \\
\hline $\mathrm{Ta}$ & $1,26 \pm 0,09$ & $1,15 \pm 0,2$ & $0,8 \pm 0,1$ & $0,8 \pm 0,2$ & $0,78 \pm 0,02$ \\
\hline Th & $1,2 \pm 0,1$ & $1,22 \pm 0,02$ & $5,9 \pm 0,5$ & $5,6 \pm 1,2$ & $5,83 \pm 0,05$ \\
\hline $\mathrm{U}$ & $0,41 \pm 0,05$ & $0,41 \pm 0,04$ & $1,7 \pm 0,1$ & $1,6 \pm 0,3$ & $1,68 \pm 0,02$ \\
\hline
\end{tabular}

Примечание: жирным шрифтом выделены концентрации железа, которое используется в качестве внутреннего стандарта.

Note: iron concentrations used as ineternal standard are in bold.

ниже предела обнаружения, в остальных стандартных образцах определено с большой погрешностью. Также получены завышенные значения концентраций по $\mathrm{Cu}$ относительно принятых значений в BCR-2, CГ-1a, CГ-3, для которых уровень содержания близок к пределу обнаружения. Остальные результаты, полученные ИСП-МС и ЛА-ИСП-МС, в пределах погрешности удовлетворительно согласуются между собой и с принятыми значениями.

На рис. 1, 2 представлено нормированное на хондрит распределение редкоземельных элементов, концентрации которых получены с помощью ИСП-МС и ЛА-ИСП-МС методик в стандартных образцах. На основании полученных результатов можно заключить, что характер распределения сохраняется, но результаты ЛА-ИСП-МС характеризуются большим разбросом по сравнению с данными, полученными в растворах.

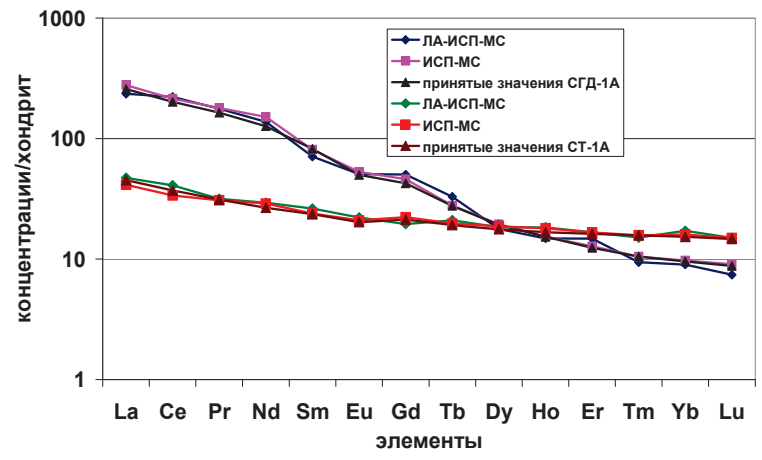

Рис.1. Нормированное распределение редкоземельных элементов в стандартных образиах СГД-1а и СТ-1А, полученное с помощью ИСП-МС и ЛА-ИСП-МС

Fig. 1. Sample/chondrite ratio of rare earth elements in reference materials SGD-1A and ST-1A obtained by ICP-MS and LA-ICP-MS 
Таблица 3. Результаты ИСП-МС и ЛА-ИСП-МС определения концентраций элементов в стандартных образиах СГД-1а и СГ-1а. Кониентрации основных элементов приведены в массовых \%, примесных элементов - в мкг/г

Table 3. Results of ICP-MS and LA-ICP-MS analyses of reference materials SGD-1a and SG-1a. Concentrations of major (wt.\%) and trace elements (ppm) are presented

\begin{tabular}{|c|c|c|c|c|c|c|}
\hline \multirow{2}{*}{$\begin{array}{l}\text { Элемент } \\
\text { Element }\end{array}$} & \multicolumn{3}{|c|}{ СГД-1a/SGD-1a (n=8) } & \multicolumn{3}{|c|}{ CГ-1a/SG-1a $(n=8)$} \\
\hline & $\begin{array}{l}\mathrm{C}_{\text {mean }} \pm \Delta \\
\text { ICP-MS }\end{array}$ & $\begin{array}{c}\mathrm{C}_{\text {mean }} \pm \Delta \\
\text { LA-ICP-MS }\end{array}$ & $\begin{array}{c}\text { Принятые значения } \\
\text { Reference value [24, 25] }\end{array}$ & $\begin{array}{l}\mathrm{C}_{\text {mean }} \pm \Delta \\
\text { ICP-MS }\end{array}$ & $\begin{array}{c}\mathrm{C}_{\text {mean }} \pm \Delta \\
\text { LA-ICP-MS }\end{array}$ & $\begin{array}{c}\text { Принятые значения } \\
\text { Reference value }[24,25]\end{array}$ \\
\hline $\mathrm{Na}$ & $(1,93 \pm 0,09) \%$ & $(2,0 \pm 0,5) \%$ & $(2,09 \pm 0,03) \%$ & $(3,6 \pm 0,4) \%$ & $(5 \pm 1) \%$ & $(4,05 \pm 0,04) \%$ \\
\hline $\mathrm{Mg}$ & $(4,2 \pm 0,2) \%$ & $(4,1 \pm 0,9) \%$ & $(4,20 \pm 0,06) \%$ & $(0,030 \pm 0,009) \%$ & $(0,010 \pm 0,007) \%$ & $(0,03 \pm 0,01) \%$ \\
\hline $\mathrm{Al}$ & $(7,89 \pm 0,4) \%$ & $(7,6 \pm 0,5) \%$ & $(7,88 \pm 0,04) \%$ & $(6,9 \pm 0,6) \%$ & $(6,8 \pm 0,7) \%$ & $(7,32 \pm 0,02) \%$ \\
\hline $\mathrm{Si}$ & $(22 \pm 1) \%$ & $(18 \pm 4) \%$ & $(21,65 \pm 0,05) \%$ & $(34 \pm 1) \%$ & $(26 \pm 7) \%$ & $(34,2 \pm 0,1) \%$ \\
\hline $\mathrm{P}$ & $(0,42 \pm 0,02) \%$ & $(0,3 \pm 0,1) \%$ & $(0,44 \pm 0,01) \%$ & $(0,008 \pm 0,002) \%$ & ниже п.о. & $(0,006 \pm 0,001) \%$ \\
\hline $\mathrm{K}$ & $(2,2 \pm 0,5) \%$ & $(2,5 \pm 0,5) \%$ & $(2,45 \pm 0,04) \%$ & $(3,3 \pm 0,4) \%$ & $(3,3 \pm 0,7) \%$ & $(3,43 \pm 0,05) \%$ \\
\hline $\mathrm{Ca}$ & $(7,8 \pm 0,4) \%$ & $(8,4 \pm 1,2) \%$ & $(7,84 \pm 0,06) \%$ & $(0,12 \pm 0,02) \%$ & $(0,07 \pm 0,03) \%$ & $(0,10 \pm 0,01) \%$ \\
\hline $\mathrm{Sc}$ & $25 \pm 3$ & $20 \pm 4$ & $24 \pm 2$ & $5 \pm 1$ & $4 \pm 1$ & $4,7 \pm 0,4$ \\
\hline $\mathrm{Ti}$ & $(1,01 \pm 0,18) \%$ & $(0,90 \pm 0,15) \%$ & $(1,03 \pm 0,02) \%$ & $(0,037 \pm 0,008) \%$ & $(0,037 \pm 0,009) \%$ & $(0,043 \pm 0,003) \%$ \\
\hline $\mathrm{V}$ & $240 \pm 30$ & $216 \pm 70$ & $250 \pm 20$ & $5,4 \pm 0,7$ & $3 \pm 2$ & $6,2 \pm 1,1$ \\
\hline $\mathrm{Cr}$ & $58 \pm 9$ & $54 \pm 16$ & $55 \pm 4$ & $9 \pm 1$ & $13 \pm 5$ & $10 \pm 1$ \\
\hline Mn & $(0,14 \pm 0,01) \%$ & $(0,13 \pm 0,03) \%$ & $(0,13 \pm 0,01) \%$ & $(0,15 \pm 0,02) \%$ & $(0,15 \pm 0,04) \%$ & $(0,15 \pm 0,01) \%$ \\
\hline $\mathrm{Fe}$ & $(7,7 \pm 0,8) \%$ & $8,2 \%$ & $(8,2 \pm 0,2) \%$ & $(1,4 \pm 0,2) \%$ & $1,56 \%$ & $(1,56 \pm 0,03) \%$ \\
\hline Co & $38 \pm 4$ & $40 \pm 10$ & $39 \pm 3$ & $0,8 \pm 0,1$ & $0,9 \pm 0,3$ & $1,0 \pm 0,3$ \\
\hline $\mathrm{Ni}$ & $42 \pm 9$ & $41 \pm 15$ & $50 \pm 5$ & $7 \pm 1$ & $9 \pm 4$ & $4,7 \pm 0,4$ \\
\hline $\mathrm{Cu}$ & $66 \pm 6$ & $51 \pm 14$ & $60 \pm 6$ & $34 \pm 4$ & $40 \pm 12$ & $27 \pm 3$ \\
\hline $\mathrm{Zn}$ & $132 \pm 10$ & $95 \pm 25$ & $120 \pm 10$ & $230 \pm 20$ & $306 \pm 80$ & $250 \pm 30$ \\
\hline $\mathrm{Ga}$ & $21 \pm 2$ & $21 \pm 4$ & $21 \pm 1$ & $38 \pm 3$ & $52 \pm 10$ & $41 \pm 2$ \\
\hline $\mathrm{Rb}$ & $70 \pm 6$ & $66 \pm 10$ & $79 \pm 3$ & $1200 \pm 100$ & $1190 \pm 200$ & $1200 \pm 100$ \\
\hline $\mathrm{Sr}$ & $2170 \pm 200$ & $2000 \pm 400$ & $2300 \pm 150$ & $3 \pm 2$ & $3 \pm 2$ & $5,6 \pm 0,6$ \\
\hline $\mathrm{Y}$ & $31 \pm 3$ & $33 \pm 6$ & $30 \pm 2$ & $74 \pm 7$ & $60 \pm 12$ & $66 \pm 3$ \\
\hline $\mathrm{Zr}$ & $230 \pm 20$ & $162 \pm 20$ & $240 \pm 20$ & $740 \pm 70$ & $600 \pm 150$ & $740 \pm 30$ \\
\hline $\mathrm{Nb}$ & $8,9 \pm 0,5$ & $9 \pm 2$ & $8,1 \pm 0,7$ & $400 \pm 40$ & $386 \pm 70$ & $400 \pm 30$ \\
\hline $\mathrm{Cs}$ & $4,1 \pm 0,5$ & н.о. & $3,2 \pm 0,2$ & $12 \pm 1$ & н.о. & $11 \pm 1$ \\
\hline $\mathrm{Ba}$ & $1280 \pm 100$ & $1060 \pm 130$ & $1200 \pm 100$ & $7 \pm 2$ & $8 \pm 4$ & $5,7 \pm 0,6$ \\
\hline $\mathrm{Hf}$ & $5 \pm 1$ & $6 \pm 1$ & $5,9 \pm 0,2$ & $36 \pm 3$ & $29 \pm 6$ & $39 \pm 4$ \\
\hline $\mathrm{Ta}$ & $0,5 \pm 0,1$ & $0,6 \pm 0,1$ & $0,7 \pm 0,1$ & $25 \pm 2$ & $26 \pm 4$ & $26 \pm 2$ \\
\hline Th & $9,4 \pm 0,9$ & $9 \pm 2$ & $9 \pm 1$ & $140 \pm 10$ & $141 \pm 25$ & $130 \pm 10$ \\
\hline $\mathrm{U}$ & $2,4 \pm 0,2$ & $2,0 \pm 0,4$ & $2,2 \pm 0,2$ & $58 \pm 4$ & $52 \pm 10$ & $63 \pm 4$ \\
\hline
\end{tabular}

Примечание: жирным шрифтом выделены концентрации железа, которое используется в качестве внутреннего стандарта.

Note: iron concentrations used as ineternal standard are in bold.

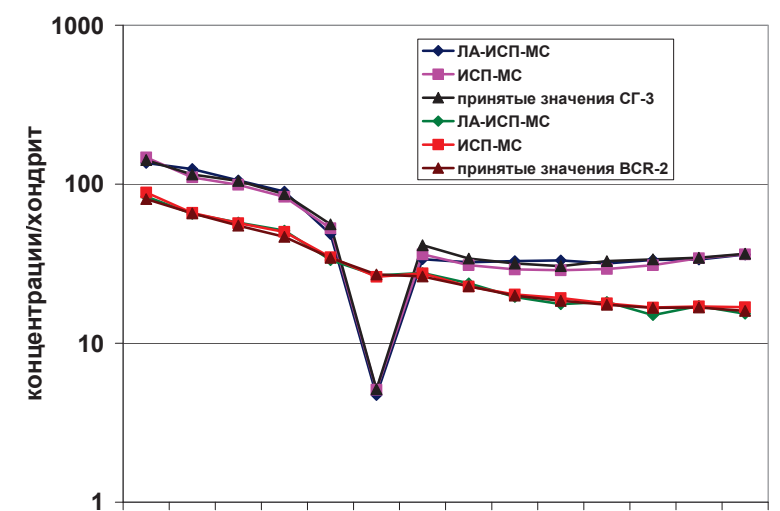

La Ce Pr Nd Sm Eu Gd Tb Dy Ho Er Tm Yb Lu элементы

Рис. 2. Нормированное распределение редкоземельных элементов в стандартных образиах $C \Gamma-3$ и BCR-2, полученное с помощью ИСП-МС и ЛА-ИСП-МС

Fig. 2. Sample/chondrite ratio of rare earth elements in reference ma terials SG-3a and BCR-2 obtained by ICP-MS and LA-ICP-MS

\section{Заключение}

Таким образом, опробованная ЛА-ИСП-МС методика многоэлементного анализа геологических образцов позволяет достоверно определять 40 элементов в готовых стёклах без дополнительной стадии пробоподготовки. Результаты, полученные для растворов и сплавленных стекол, с использованием градуировок по стандартным геологическим образцам BHVO-1 и BHVO-2, в пределах погрешности согласуются между собой и с принятыми значениями. Методика ЛА-ИСП-МС отличается от методики ИСП-МС экспрессностью за счет отсутствия стадии пробоподготовки, пределы обнаружения и погрешность определения для ЛА-ИСП-МС методики выше, чем при анализе растворов, что ограничивает ее применение для определения некоторых элементов.

Работа выполнена по государственному заданию ИГМ СО РАН. 
Таблица 4. Результаты ИСП-МС и ЛА-ИСП-МС определения кониентраций элементов в стандартных образцах СТ-1а и СГ-з. Кониентрации основных элементов приведены в массовых \%, примесных элелентов - в мкг/г

Table 4. Results of ICP-MS and LA-ICP-MS analyses of reference materials ST-1 a and SG-3. Concentrations of major (wt. \%) and trace elements (ppm) are presented

\begin{tabular}{|c|c|c|c|c|c|c|}
\hline \multirow{2}{*}{$\begin{array}{l}\text { Элемент } \\
\text { Element }\end{array}$} & \multicolumn{3}{|c|}{ CT-1a/ST-1a $(n=8)$} & \multicolumn{3}{|c|}{ CГ-3/SG-3 (n=8) } \\
\hline & $\begin{array}{l}\mathrm{C}_{\text {mean }} \pm \Delta \\
\text { ICP-MS }\end{array}$ & $\begin{array}{c}\mathrm{C}_{\text {mean }} \pm \Delta \\
\text { LA-ICP-MS }\end{array}$ & $\begin{array}{c}\text { Принятые значения } \\
\text { Reference value [24, 25] }\end{array}$ & $\begin{array}{l}\mathrm{C}_{\text {mean }} \pm \Delta \\
\text { ICP-MS }\end{array}$ & $\begin{array}{c}\mathrm{C}_{\text {mean }} \pm \Delta \\
\text { LA-ICP-MS }\end{array}$ & $\begin{array}{c}\text { Принятые значения } \\
\text { Reference value [24, 25] }\end{array}$ \\
\hline $\mathrm{Na}$ & $(1,8 \pm 0,1) \%$ & $(1,9 \pm 0,4) \%$ & $(1,85 \pm 0,02) \%$ & $(2,9 \pm 0,2) \%$ & $(3,5 \pm 0,9) \%$ & $(3,15 \pm 0,04) \%$ \\
\hline $\mathrm{Mg}$ & $(3,7 \pm 0,3) \%$ & $(3,5 \pm 0,8) \%$ & $(3,44 \pm 0,04) \%$ & $(0,06 \pm 0,006) \%$ & $(0,05 \pm 0,01) \%$ & $(0,06 \pm 0,01) \%$ \\
\hline $\mathrm{Al}$ & $(7,46 \pm 0,07) \%$ & $(7,7 \pm 0,3) \%$ & $(7,53 \pm 0,05) \%$ & $(5,3 \pm 0,3) \%$ & $5,0 \pm 0,8$ & $(5,63 \pm 0,04) \%$ \\
\hline $\mathrm{Si}$ & $(23 \pm 2) \%$ & $(24 \pm 4) \%$ & $(22,91 \pm 0,05) \%$ & $(37 \pm 3) \%$ & $(37 \pm 6) \%$ & $(34,89 \pm 0,07) \%$ \\
\hline $\mathrm{P}$ & $(0,09 \pm 0,01) \%$ & $(0,11 \pm 0,05) \%$ & $(0,092 \pm 0,004) \%$ & $(0,011 \pm 0,005) \%$ & ниже п.о. & $(0,011 \pm 0,003) \%$ \\
\hline $\mathrm{K}$ & $(0,53 \pm 0,05) \%$ & $(0,56 \pm 0,09) \%$ & $(0,58 \pm 0,08) \%$ & $(3,9 \pm 0,3) \%$ & $(3,2 \pm 0,7) \%$ & $(3,85 \pm 0,05) \%$ \\
\hline $\mathrm{Ca}$ & $(7,4 \pm 0,4) \%$ & $(7 \pm 1) \%$ & $(7,29 \pm 0,07) \%$ & $(0,22 \pm 0,02) \%$ & $(0,18 \pm 0,06) \%$ & $(0,23 \pm 0,02) \%$ \\
\hline Sc & $39 \pm 3$ & $36 \pm 8$ & $37 \pm 3$ & $4,0 \pm 0,5$ & $3 \pm 1$ & $4,2 \pm 0,2$ \\
\hline $\mathrm{Ti}$ & $(1,08 \pm 0,08) \%$ & $(1,0 \pm 0,2) \%$ & $(1,11 \pm 0,02) \%$ & $(0,16 \pm 0,01) \%$ & $(0,13 \pm 0,02) \%$ & $(0,156 \pm 0,006) \%$ \\
\hline $\mathrm{V}$ & $322 \pm 30$ & $330 \pm 60$ & $310 \pm 30$ & $4,3 \pm 0,4$ & $5 \pm 1$ & $4,9 \pm 0,8$ \\
\hline $\mathrm{Cr}$ & $152 \pm 10$ & $158 \pm 50$ & $140 \pm 10$ & $34 \pm 3$ & $33 \pm 9$ & $34 \pm 1$ \\
\hline $\mathrm{Mn}$ & $(0,19 \pm 0,03) \%$ & $(0,17 \pm 0,04) \%$ & $(0,160 \pm 0,008) \%$ & $(0,10 \pm 0,01) \%$ & $(0,10 \pm 0,03) \%$ & $(0,091 \pm 0,004) \%$ \\
\hline $\mathrm{Fe}$ & $(9,3 \pm 0,9) \%$ & $10,6 \%$ & $(10,6 \pm 0,1) \%$ & $(2,9 \pm 0,3) \%$ & $3,15 \%$ & $(3,15 \pm 0,05) \%$ \\
\hline Co & $46 \pm 8$ & $49 \pm 10$ & $45 \pm 4$ & $0,82 \pm 0,07$ & $0,9 \pm 0,3$ & $0,75 \pm 0,06$ \\
\hline $\mathrm{Ni}$ & $80 \pm 8$ & $84 \pm 20$ & $90 \pm 6$ & $4 \pm 3$ & $7 \pm 4$ & $5,8 \pm 0,6$ \\
\hline $\mathrm{Cu}$ & $193 \pm 10$ & $190 \pm 40$ & $180 \pm 20$ & $13 \pm 1$ & $30 \pm 10$ & $13 \pm 1$ \\
\hline $\mathrm{Zn}$ & $125 \pm 10$ & $130 \pm 30$ & $140 \pm 20$ & $140 \pm 10$ & $160 \pm 40$ & $134 \pm 7$ \\
\hline $\mathrm{Ga}$ & $20 \pm 1$ & $20 \pm 4$ & $19 \pm 1$ & $27 \pm 2$ & $30 \pm 6$ & $27 \pm 1$ \\
\hline $\mathrm{Rb}$ & $17 \pm 4$ & $17 \pm 3$ & $17 \pm 1$ & $127 \pm 10$ & $149 \pm 25$ & $130 \pm 6$ \\
\hline $\mathrm{Sr}$ & $228 \pm 20$ & $206 \pm 40$ & $230 \pm 10$ & $8,0 \pm 0,8$ & $7 \pm 2$ & $7,2 \pm 0,7$ \\
\hline $\mathrm{Y}$ & $35 \pm 4$ & $35 \pm 7$ & $32 \pm 3$ & $59 \pm 5$ & $57 \pm 12$ & $57 \pm 2$ \\
\hline $\mathrm{Zr}$ & $140 \pm 15$ & $127 \pm 20$ & $150 \pm 30$ & $500 \pm 50$ & $444 \pm 70$ & $520 \pm 20$ \\
\hline $\mathrm{Nb}$ & $8,0 \pm 0,8$ & $6,7 \pm 0,9$ & $7,2 \pm 0,6$ & $19 \pm 2$ & $22 \pm 4$ & $19 \pm 1$ \\
\hline Cs & $0,7 \pm 0,2$ & н.о. & 0,9 & $4,1 \pm 0,4$ & н.о. & 4,5 \\
\hline $\mathrm{Ba}$ & $231 \pm 20$ & $215 \pm 25$ & $210 \pm 10$ & $71,0 \pm 0,9$ & $63 \pm 10$ & $76 \pm 5$ \\
\hline $\mathrm{Hf}$ & $3,3 \pm 0,3$ & $3,2 \pm 0,7$ & $3,3 \pm 0,6$ & $11 \pm 1$ & $11 \pm 3$ & $12 \pm 1$ \\
\hline $\mathrm{Ta}$ & $0,6 \pm 0,1$ & $0,9 \pm 0,1$ & $0,9 \pm 0,3$ & $1,2 \pm 0,1$ & $1,4 \pm 0,2$ & $1,4 \pm 0,1$ \\
\hline Th & $1,7 \pm 0,1$ & $1,4 \pm 0,5$ & $2,3 \pm 0,5$ & $7,4 \pm 0,9$ & $7,2 \pm 1,3$ & $7,6 \pm 0,6$ \\
\hline $\mathrm{U}$ & $0,7 \pm 0,1$ & $0,5 \pm 0,2$ & $0,8 \pm 0,2$ & $2,2 \pm 0,2$ & $2,7 \pm 0,7$ & $1,9 \pm 0,1$ \\
\hline
\end{tabular}

Примечание: жирным шрифтом выделены кониентрации железа, которое используется в качестве внутреннего стандарта.

Note: iron concentrations used as ineternal standard are in bold.

\section{СПИСОК ЛИТЕРАТУРЫ}

1. ICP-MS - a powerful tool for high-precision trace-element analysis in Earth sciences: Evidence from analysis of selected U.S.G.S. reference samples / G.A. Jenner, H.P. Longerich, S.E. Jackson, B.J. Freyer // Chemical Geology - 1990 - V. 83. - P. 133-148.

2. Balaram V. Recent trends in the instrumental analysis of rare earth elements in geological and industrial materials // Trends in Analytical Chemistry. - 1996. - V. 15. - P. 475-485.

3. Jarvis K.E., Williams J.G. Laser ablation inductively coupled plasma mass spectrometry (LA-ICP-MS): a rapid technique for the direct, quantitative determination of major, trace and rare-earth elements in geological samples // Chemical Geology. - 1993. V. 106. - P. 251-262.

4. Пупышев А.А., Суриков В.Т. Масс-спектрометрия с индуктивно связанной плазмой. Образование ионов. - Екатеринбург: Уp0 PAH, 2006. - 276 c.

5. Jenner F.E., Arevalo R.D. Major and trace element analysis of natural and experimental igneous systems using LA-ICP-MS // Elements. - 2016. - V. 12. - № 5. - P. 311-316.

6. Petrelli M., Laeger K., Perugini D. High spatial resolution trace element determination of geological samples by laser ablation quadrupole plasma mass spectrometry: implications for glass analy- sis in volcanic products / Geosciences Journal. - 2016. - V. 20. № 6. - P. 851-863.

7. Geochronological, geochemical and mineralogical constrains on the petrogenesis of appinites from the Laoniushan complex, eastern Qinling, central China / L. Ding, C. Ma, J. Li, L. Wang // Chemie der Erde. - 2016. - V. 76. - № 4. - P. 579-595.

8. Evaluating downhole fractionation corrections in LA-ICP-MS U$\mathrm{Pb}$ zircon geochronology / T.J. ver Hoeve, J.S. Scoates, C.J. Wall, D. Weis, M. Amini // Chemical Geology. - 2018. № 483. - P. 201-217.

9. Eggins S.M. Laser Ablation ICP-MS Analysis of Geological Materials Prepared as Lithium Borate Glasses // Geostandarts Newsletter. - 2003. - V. 27. - № 2. - P. 147-162.

10. Zongshou Y., Norman M.D., Robinson P. Major and Trace Element Analysis of Silicate Rocks by XRF and Laser Ablation ICP-MS Using Lithium Borate Fused Glasses: Matrix Effects, Instrument Response and Results for International Reference Materials // Geostandarts Newsletter. - 2002. - V. 27. - № 1. - P. 67-89.

11. Orihashi Y., Takafumi H. Rapid quantitative analysis of $Y$ and REE abundances in XRF glass bead for selected GSJ reference rock standards using Nd-YAG $266 \mathrm{~nm}$ UV laser ablation ICP-MS // Geochemical Journal. - 2003. - V. 37. - P. 401-412. 
12. Elemental Analyses Using Laser Ablation-Inductively Coupled Plasma-Mass Spectrometry (LA-ICP-MS) of Geological Samples Fused with $\mathrm{Li}_{2} \mathrm{~B}_{4} \mathrm{O}_{7}$ and Calibrated Without Matrix-Matched Standards / D. Guenther, A. Quadt, R. Wirz, H. Cousin, V.J. Dietrich // Mikrochimica Acta. - 2001. - V. 136. - P. 101-107.

13. Application of a double-focusing magnetic sector inductively coupled plasma mass spectrometer with laser ablation for the bulk analysis of rare earth elements in rocks fused with $\mathrm{Li}_{2} \mathrm{~B}_{4} \mathrm{O}_{7}$ M. Ødegård, S.H. Dundas, B. Flem, A. Grimstvedt // Fresenius Journal of Analytical Chemistry. - 1998. - V. 362. - P. 477-482.

14. Weis P., Beck H.P., Guenther D. Characterizing ablation and aerosol generation during elemental fractionation on absorption modified lithium tetraborate glasses using LA-ICP-MS // Anal Bioanal Chem. - 2005. - V. 381. - P. 212-224.

15. Trace Element Analysis of Fused Whole-Rock Glasses by Laser Ablation-ICP-MS and PIXE / M. Kurosawa, K. Shima, S. Ishii, K. Sasa // Geostandarts and Geoanalytical Research. - 2005. V. 30. - № 1. - P. 17-30

16. Sylvester P.J. Trace Element Analysis of Fused Whole Rock Glass by Laser Ablation ICPMS // Laser-Ablation-ICPMS in the Earth Sciences. - 2001. - V. 29. - P. 147-162.

17. Laser-ablation ICP-MS analysis of siliceous rock glasses fused on an iridium strip heater using $\mathrm{MgO}$ dilution / F. Nehring, D.E. Jacob, M.G. Barth, S.F. Foley // Mikrochimica Acta. - 2008. V. 160 - - P. 153-163.

18. A Flux-Free Fusion Technique for Rapid Determination of Major and Trace Elements in Silicate Rocks by LA-ICP-MS / Z. He, F. Huang, H. Yu, Y. Xiao, F. Wang, Q. Li, Y. Xia, X. Zhang // Geostandards and Geoanalytical Research. - 2016 - V. 40. № 1. - P. 5-27

19. Calibration and correction of LA-ICP-MS and LA-MC-ICP-MS analyses for element contents and isotopic ratios / J. Lin, Y. Liu,
Y. Yang, Z. Hu // Solid Earth Sciences. - 2016. - V. 1. - № 1. P. 5-27.

20. Определение редкоземельных и высокозарядных элементов в стандартных геологических образцах методом масс-спектрометрии с индуктивно-связанной плазмой (ИСП-МС) / И.В. Николаева, С.В. Палесский, О.А. Козьменко, Г.Н. Аношин // Геохимия. - 2008. - № 10. - С. 1085-1091.

21. Карманова Н.Г., Карманов Н.С. Универсальная методика рентгенофлуоресцентного силикатного анализа горных пород на спектрометре ARL-9900XP // Тезисы докладов VII всероссийской конференции по рентгеноспектральному анализу. Новосибирск, 2011. - С. 126.

22. Определение основных и примесных элементов в силикатных породах методом масс-спектрометрии с индуктивно-связанной плазмой после сплавления с $\mathrm{LiBO}_{2}$ / И.В. Николаева, С.В. Палесский, О.С. Чирко, С.М. Черноножкин // Аналитика и контроль. - 2012. - Т. 16. - № 2. - С. 134-142.

23. GeoReM: a New Geochemical Database for Reference Materials and Isotopic Standards / K.P. Jochum, U. Nohl, K. Herwig, E. Lammel, D. Stoll, A.W. Hofmann. URL: http://georem.mpchmainz.gwdg.de (дата обращения 20.03.2018).

24. Новые данные по определению редких и рассеянных элементов в геологических стандартных образцах методом масс-спектрометрии с индуктивно-связанной плазмой / И.Н. Мысовская, Е.В. Смирнова, В.И. Ложкин, Н.Н. Пахомова / Заводская лаборатория. Диагностика материалов. - 2009. - № 10. C. $60-66$

25. Петров Л.Л. Каталог - Стандартные образцы химического состава природных минеральных веществ. - Иркутск. 2006. $54 \mathrm{c.}$

Поступила 08.06.2018 г.

\section{Информация об авторах}

Николаева И.В., кандидат химических наук, старший научный сотрудник Института геологии и минералогии им. В.С. Соболева СО РАН.

Палесский C.B., кандидат химических наук, старший научный сотрудник Института геологии и минералогии им. В.С. Соболева СО РАН.

Kapnoв A.B., аспирант Института геологии и минералогии им. В.С. Соболева СО РАН. 
UDC 550.4.08

\section{ANALYSIS OF GEOLOGICAL SAMPLES: COMPARISON OF SOLUTION ICP-MS AND FUSED GLASSES LA-ICP-MS TECHNIQUES}

Irina V. Nikolaeva',

inikol@igm.nsc.ru

Stanislav V. Palesskiy',

stas@igm.nsc.ru

Alexander V. Karpov',

theblake@mail.ru

1 V.S. Sobolev Institute of geology and mineralogy SB RAS,

3, Akademik Koptyug avenue, Novosibirsk, 630090, Russia.

The relevance of the study is caused by the need to develop new efficient sample preparation technique suitable for routine multielement ICP-MS analysis of geological samples which decreases time consumption and allows determining a wide number of elements in the range of single measurement using laser ablation (LA-ICP-MS).

The main aim of the study is to compare metrological characteristics of two ICP-MS techniques, based on different sample preparation of silicate rocks - sample dissolution after melting with lithium metaborate and using prepared fused glasses for laser ablation.

Objects: the international and Russian reference materials of natural rocks: basalts - BHVO-1, BHVO-2, BCR-2, granites - SG-1a, SG-3, gabbro SGD-1a, trap ST-1a.

Methods: inductively coupled plasma mass spectrometry (ICP-MS) and laser ablation inductively coupled plasma mass spectrometry (LA-ICP-MS).

Results of the study showed that the developed multielement ICP-MS and LA-ICP-MS techniques allow determining a wide number of elements in geological samples in the range of single measurement using low, medium and high resolution. Internal and matrix-matched external standards for decreasing/correction of matrix effects and instrumental drift are used. Comparison of the obtained results with values of six reference geological materials verified the accuracy of the techniques. The LA-ICP-MS technique is faster due to the lack of sample preparation, but has higher uncertainty and detection limits in comparison to the ICP-MS technique.

\section{Key words:}

Inductively coupled plasma mass spectrometry (ICP-MS), laser ablation (LA), sample preparation, geological reference materials, major and trace elements, rare earth elements.

The research was carried out by the State Task of IGM SB RAS.

\section{REFERENCES}

1. Jenner G.A., Longerich H.P., Jackson S.E., Freyer B.J. ICP-MS a powerful tool for high-precision trace-element analysis in Earth sciences: Evidence from analysis of selected U.S.G.S. reference samples. Chemical Geology, 1990, vol. 83, pp. 133-148.

2. Balaram V. Recent trends in the instrumental analysis of rare earth elements in geological and industrial materials. Trends in Analytical Chemistry, 1996, vol. 15, pp. 475-485.

3. Jarvis K.E., Williams J.G. Laser ablation inductively coupled plasma mass spectrometry (LA-ICP-MS): a rapid technique for the direct, quantitative determination of major, trace and rare-earth elements in geological samples. Chemical Geology, 1993, vol. 106, pp. 251-262.

4. Pupyshev A.A. Mass-spektrometriya s induktivno-suyazannoy plazmoy. Obrazovanie ionov [Inductively coupled plasma massspectrometry. Generation of ions]. Ekaterinburg, UrO RAS Publ., 2006. 276 p.

5. Jenner F.E., Arevalo R.D. Major and trace element analysis of natural and experimental igneous systems using LA-ICP-MS. Elements, 2016, vol. 12, no. 5, pp. 311-316.

6. Petrelli M., Laeger K., Perugini D. High spatial resolution trace element determination of geological samples by laser ablation quadrupole plasma mass spectrometry: implications for glass analysis in volcanic products. Geosciences Journal, 2016, vol. 20, no. 6 , pp. 851-863.

7. Ding L., Ma C., Li J., Wang L. Geochronological, geochemical and mineralogical constrains on the petrogenesis of appinites from the Laoniushan complex, eastern Qinling, central China. Chemie der Erde, 2016, vol. 76, no. 4, pp. 579-595.

8. Ver Hoeve T.J., Scoates J.S., Wall C.J., Weis D., Amini M. Evaluating downhole fractionation corrections in LA-ICP-MS U-Pb zircon geochronology. Chemical Geology, 2018, no. 483, pp. 201-217.

9. Eggins S.M. Laser Ablation ICP-MS Analysis of Geological Materials Prepared as Lithium Borate Glasses. Geostandarts Newsletter, 2003, vol. 27, no. 2, pp. 147-162.

10. Zongshou Y., Norman M.D., Robinson P. Major and Trace Element Analysis of Silicate Rocks by XRF and Laser Ablation ICPMS Using Lithium Borate Fused Glasses: Matrix Effects, Instrument Response and Results for International Reference Materials. Geostandarts Newsletter, 2002, vol. 27, no. 1, pp. 67-89.

11. Orihashi Y., Takafumi H. Rapid quantitative analysis of $Y$ and REE abundances in XRF glass bead for selected GSJ reference rock standards using Nd-YAG $266 \mathrm{~nm}$ UV laser ablation ICP-MS. Geochemical Journal, 2003, vol. 37, pp. 401-412.

12. Guenther D., Quadt A., Wirz R., Cousin H., Dietrich V.J. Elemental Analyses Using Laser Ablation-Inductively Coupled Plasma-Mass Spectrometry (LA-ICP-MS) of Geological Samples Fused with $\mathrm{Li}_{2} \mathrm{~B}_{4} \mathrm{O}_{7}$ and Calibrated Without Matrix-Matched Standards. Mikrochimica Acta, 2001, vol. 136, pp. 101-107.

13. Ødegård M., Dundas S.H., Flem B., Grimstvedt A. Application of a double-focusing magnetic sector inductively coupled plasma mass spectrometer with laser ablation for the bulk analysis of rare earth elements in rocks fused with $\mathrm{Li}_{2} \mathrm{~B}_{4} \mathrm{O}_{7}$. Fresenius Journal of Analytical Chemistry, 1998, vol. 362, pp. 477-482. 
14. Weis P., Beck H.P., Guenther D. Characterizing ablation and aerosol generation during elemental fractionation on absorption modified lithium tetraborate glasses using LA-ICP-MS. Anal Bioanal Chem., 2005, vol. 381, pp. 212-224.

15. Kurosawa M., Shima K., Ishii S., Sasa K. Trace Element Analysis of Fused Whole-Rock Glasses by Laser Ablation-ICP-MS and PIXE. Geostandarts and Geoanalytical Research, 2005, vol. 30, no. 1 , pp. 17-30.

16. Sylvester P.J. Trace Element Analysis of Fused Whole Rock Glass by Laser Ablation ICPMS. Laser-Ablation-ICPMS in the Earth Sciences, 2001, vol. 29, pp. 147-162.

17. Nehring F., Jacob D.E., Barth M.G., Foley S.F. Laser-ablation ICP-MS analysis of siliceous rock glasses fused on an iridium strip heater using $\mathrm{Mg} 0$ dilution. Mikrochimica Acta, 2008, vol. 160, pp. $153-163$.

18. He Z., Huang F., Yu H., Xiao Y., Wang F., Li Q., Xia Y., Zhang X. A Flux-Free Fusion Technique for Rapid Determination of Major and Trace Elements in Silicate Rocks by LA-ICP-MS. Geostandards and Geoanalytical Research, 2016 March, vol. 40, no. 1, pp. 5-27.

19. Lin J., Liu Y., Yang Y., Hu. Z Calibration and correction of LAICP-MS and LA-MC-ICP-MS analyses for element contents and isotopic ratios. Solid Earth Sciences, 2016, vol. 1, no. 1, pp. 5-27.

20. Nikolaeva I.V., Palesskiy S.V., Kozmenko O.A., Anoshin G.N. Opredelenie redkozemelnykh i vysokozaryadnykh elementov v standartnykh geologicheskykh obraztsakh metodom mass-spektrometrii s induktivno-svyazannoy plazmoy [Determination of rare earth and high-charged elements in standard materials using inductively coupled plasma mass-spectrometry (ICP-MS)]. Geochemistry, 2008, no. 10, pp. 1085-1091.
21. Karmanova N.G., Karmanov N.S. Universalnaya metodika rentgenofluorestsentnogo silikatnogo analiza gornykh porod na spektrometre ARL-9900XP [Universal X-ray fluorescence technique for silicate analysis of rocks using ARL-9900XP spectrometer]. Tezisy dokladov VII vserossiyskoy konferentsii po rentgenospektralnomu analizu [Proc. of the VII All-Russian X-ray spectral analysis conference]. Novosibirsk, 2011. p. 126.

22. Nikolaeva I.V., Palesskiy S.V., Chirko O.S., Chernonozhkin S.M. Opredelenie osnovnykh i primesnykh elementov v silikatnykh porodakh metodom mass-spektrometrii s induktivno-svyazannoy plazmoy posle splavleniya $\mathrm{s} \mathrm{LiBO}_{2}$ [Determination of major and trace elements in silicate rocks by inductively couples plasma mass-spectrometry after fusion with $\mathrm{LiBO}_{2}$ ]. Analytics and control, 2012, vol. 16, no. 2, pp.134-142.

23. Jochum K.P., Nohl U., Herwig K., Lammel E., Stoll D., Hofmann A.W. GeoReM: a New Geochemical Database for Reference Materials and Isotopic Standards. Available at: http://georem.mpch-mainz.gwdg.de (accessed 20 March 2018).

24. Mysovskaya I.N., Smirnova E.V., Lozhkin V.I., Pakhomova N.N. Novye dannye po opredeleniyu redkikh i rasseyannykh elementov v geologicheskikh standartnykh obraztsakh metodom mass-spektrometrii s induktivno-svyazannoy plazmoy [New data on determination of rare and scattered elements in standard geological materials using inductively coupled plasma mass-spectrometry technique]. Factory laboratory. Diagnostics of materials, 2009, no. 10 , pp. 60-66.

25. Petrov L.L. Katalog - Standartnye obraztsy khimicheskogo sostava prirodnykh mineralnykh veshchestv [Catalog - Reference materials of the chemical composition of natural minerals]. Irkutsk, 2006. $54 \mathrm{p}$.

Received: 8 June 2018.

\section{Information about the authors}

Irina V. Nikolaeva, Cand. Sc., senior researcher, V.S. Sobolev Institute of geology and mineralogy SB RAS.

Stanislav V. Palesskiy, Cand. Sc., senior researcher, V.S. Sobolev Institute of geology and mineralogy SB RAS.

Alexander V. Karpov, postgraduate student, V.S. Sobolev Institute of geology and mineralogy SB RAS. 